\title{
Gnrh Agoist Uzun Protokol Tedavi Uygulanan Polikistik Over Sendromu Olan ve Olmayan Hastaların Gebelik Sonuçlarının Karşılaştırılması
}

\author{
To Compare Pregnancy Results of Patients with Or Without PcosWho Are Included \\ Gnrh Agonist Long Protocol Treatment
}

Alper SEYHAN ${ }^{1}$, Kenan SOFUOĞLU ${ }^{2}$, Merve Begüm OSMANLIOĞLU ${ }^{3}$

1. Tekirdağ Devlet Hastanesi, Jinekolojik Onkoloji Cerrahisi Bölümü, Tekirdağ, Türkiye

2. İstanbul Zeynep Kamil Kadın ve Çocuk Hastalıkları Sağlık Uygulama ve Araştırma Merkezi, İstanbul, Türkiye

3. İstanbul Medipol Üniversitesi, İstanbul, Türkiye

\section{$\ddot{O Z Z E T}$}

Kadın infertilitesinin en s1k nedeni ovulasyon bozukluklarıdır. Anovulatuar infertilitenin en temel nedeni Polikistik Over Sendromudur. Polikistik Over Sendromu fertil çağdaki kadınlarda görülen en sık endokrin bozukluktur. Polikistik Over Sendromlu infertil kiside ilk tercih ovulasyon indüksiyon ajanı Klomifen Sitrat'tır. Klomifen Sitrat'a yanıtsız olgularda eksojen gonadotropinler kullanılmaktadır. Çalıșmamızda, GnRH Agonist Uzun Protokol uygulanan PKOS olan ve olmayan hastalarda gebelik sonuçları ve yanısıra kullanılan gonadotropin dozlar, bazal FSH, E2, prolaktin değerleri, elde edilen matur oosit sayıları gibi parametreleri retrospektif olarak karşılaştırmayı hedefledik.

Anahtar Kelimeler: polikistik over sendromu,gnrh agonist uzun protokol

\section{ABSTRACT}

Ovulation defects are the most common cause of woman infertility. Polycystic Ovary Syndrome is main cause of anovulatory infertility and most commonly seen endocrine pathology in fertile period of women. In infertile patients with PCOS, first choice of induction agent is Klomifen Citrate. Patients who do not response to Klomifen Citrate, exogen gonadotropines are used for induction. In our study we aimed to evaluate gonadotrophine doses, basaline FSH, E2, prolactine levels, mature oocyte number and pregnancy rates in GnRH Agonist Long Protocol treatment in patients who have PCOS or not.

Keywords: polycystic ovary syndrome,Gnrh agonist long protocol

\section{GİRIŞ}

İnfertilite şikayetiyle başvuran kadınların \%40'inda başlıca neden ovulasyon bozukluğudur. Anovulatuvar infertilitenin en sik nedeni (\%73), Polikistik over sendromu (PKOS)'dur. Polikistik over sendromu (PKOS) fertil çağindaki kadınlarda en sık görülen endokrin bozukluktur. Sendromun siklıği yaklaşı \% \%-8 olarak bildirilmektedir (1).

İnfertil hastalarda öncelikli tedavi şemasında, düşük maliyetli girișimsel olmayan tedavi seçenekleriyle başlamak ve cevap alınmazsa girişimsel

\section{İletişim}

Sorumlu Yazar: Alper SEYHAN

Adres: Tekirdağ Devlet Hastanesi, Jinekolojik Onkoloji Cerrahisi Uzmanı, Tekirdağ, Türkiye

Tel: +90 (533) 7256466

E-Posta: alperseyhan@yahoo.com

Makale Geliş: 08.09.2019

Makale Kabul: 07.02.2020

DOI: http://dx.doi.org/10.16948/zktipb.616816 medikal ve cerrahi seçeneklerin kullanılması uygun olmaktadır (2). Polikistik over sendrom'lu olgularda ovulasyon indüksiyonu halen tedavi sürecinde ilk başvurulacak yöntemdir. Ovulasyon indüksiyonunda ilk tercih ajan, klomifen sitrat (KS)'tır (3). KS ile olguların \%80'inde ovulasyon, \%40'ında ise gebelik başarısı sağlanmaktadır. Fakat hastaların yaklaşık \%20-30'unda KS kullanımıyla ovulasyon ve / veya gebelik elde edilemez. Bu hasta grubunda eksojen gonadotropinler alternatif seçenekler olarak akilda tutulur.

Klomifen Sitrat'a yanıtsız PKOS olgularında ovulasyon indüksiyonu amaciyla eksojen gonadotropinler öncelikli olarak kullanılmaktadır (4). Günümüzde bu amaçla kullanılan gonadotropinler; Human menapozal gonadotropin (HMG), Üriner follikül stimulan hormon (u-FSH), Rekombinant follikül stimulan hormon (r-FSH) 'dur.

$\mathrm{Bu}$ genel bilgiler 1şı̆̆ında; tasarlanan çalışmamizda, Gnrh agonist uzun protokol uygulanan PKOS olan ve olmayan hastalarda gebelik sonuçlar1 ve yanısira kullanılan gonadotropin dozları, bazal FSH, E2, prolaktin değerleri, elde edilen matur oosit sayıları gibi parametreleri retrospektif olarak karş1laştırmayı hedefledik.

\section{GEREÇ ve YÖNTEM}

Bu çalışma Zeynep Kamil Eğitim ve Araştırma Hastanesi İnfertilite ve Yardımcı Üreme Teknikleri Merkezi'ne Ocak-2012 ile Aralık-2013 tarihleri arasında başvuran infertil hastalar arasından seçim ve dişlanma kriterleri belirlenen Gnrh-agonist uzun protokol uygulanmış polikistik over sendromu olan ve olmayan hastaların gebelik sonuçlarının karşılaştırılmasi için tasarlanmıştır. Kriterlere uygun hastalar retrospektif olarak değerlendirilmiştir. Öncesinde Zeynep Kamil Hastanesi bünyesinde bulunan etik kurul onayı alınmıştır. Hasta seçim kriterlerine bakılırsa ; 23-39 yaş aralığında, daha önce iki kez gonadotropinlerle ovulasyon indüksiyonu denenmiş ve yanıt alınamamış olması, adetin 3. günü $\mathrm{FSH}<$ $10 \mathrm{ugr} / \mathrm{mlt}$ olması, herhangi bir sistemik hastalı̆̆ bulunmaması, organik nedenli infertilite sebeplerinin dışlanması, hastaların sigara içmiyor olması şeklinde belirlenmiştir. Siklus iptal kriterleri ; ovulasyon indüksiyonu sonrası $>18 \mathrm{~mm}$ follikül bulunmamas1, iki kontrol süresi arası östrojen düzeyinin $>\% 50$ düşmesi, muhtemel OHSS gelişim durumu (Stimulasyonun 8. gününde 15 veya daha fazla sa- 
y1da intermediate folikül (12-16 mm) ya da 10. gün veya daha sonra, 20 veya daha fazla sayıda büyük folikül (16-20 mm) oluşumu ve/veya östrodiol konsantrasyonu $\geq 3000 \mathrm{pg} / \mathrm{ml}$ ) varlığ 1 olarak belirlenmiştir.

\section{HASTALARIN DEĞERLENDİRILMESI}

Seçilen hastaların ilk muayenelerinde obstetrik ve jinekolojik özgeçmişleri, menstruel siklus düzenleri sorguland1. Fizik muayenelerinde kan basınc1, boy, ağırlık ve VKİ (ağırlık / boy ${ }^{2}$ ) hesaplanıp kaydedildi. Pelvik muayeneleri yapıldı. Açlik kan şekeri, üre, SGOT, SGPT, Tiroid fonksiyon testleri, Erken foliküler fazda (2-3. gün) ise; bazal USG yapıld1 ve uterus boyutları, endometrium kalınlığ1, over boyutları, folikül sayısı ve çapları ölçüldü. Bunun için GE Medikal (Logic alfa 200 GE Medical A/S Milwaukee, Amerika Birleşik Devletleri) marka Ultrasonografi cihazı kullanıldı. Bazal (3. gün) FSH, LH, E2, Prolaktin değerleri ölçüldü.

Değerlendirmeyle birlikte, Gnrh-agonist uzun protokol uygulanması kararı verilen hastalara tedavinin yap1lacağ1 siklusun öncesinde midluteal fazda (19-21. Gün) Gnrh analog olarak triptorelin 0.1 $\mathrm{mg} / \mathrm{g}$ veya leuprolid asetat $500 \mathrm{mg} / \mathrm{g}$ dozunda başland1. Adetin 3. günü ya da agonist kullanımının 14 . Günü hasta control için çağırıldı. Kontrolde overlerde kist olup olmadığ́ ve E2 düzeyi değerlendirildi. $<10 \mathrm{~mm}$ kist varlığ $1 \mathrm{ve} / \mathrm{veya}$ E2 düzeyi $<50 \mathrm{pg} / \mathrm{mlt}$ düzeyleri baskılama için yeterli görüldü. Analog dozu yarıya düşürülerek gonadotropin stimulasyonuna başlandı. En az 3 adet $>17 \mathrm{~mm}$ follikül görülünce ovitrelle 250 ugr şeklinde hcg dozu yapıldı. 35-36 saat sonrasında OPU yapıld1. Embriyo transferi ise 2., 3., ya da 5. gün yap1ld1. İşlem sonras1 12 . günde b-hcg kontrolü yapıld1.

\section{BULGULAR}

Çalışmamız Ocak-2012 ile Aralık-2013 tarihleri arasında Zeynep Kamil Eğitim ve Araştırma Hastanesi İnfertilite ve Tüp bebek Merkezine başvuran, gerekli incelemeleri yap1larak tedavi uygulanmasına karar verilmiş 1756 infertilite kliniği hastasının dosyalarının incelenmesiyle başlamıştır. Yapılan retrospektif dosya taraması sonucu 321 hastaya klomifen sitrat ya da eksojen gonadotropinlerle birlikte IUI uygulaması yapılmıştır. İncelemeler sonucu 425 çiftte infertilite sebebi olarak erkek faktörü saptanmıştır. 367 çiftte yetersiz over rezervi, infertilite ya da başka sebeple geçirilmiş pelvik cerrahi öyküsü saptanmıştır. 21 hastada endometriozis tanısı konmuş, 56 çift tedavi sürecine devam etmemiştir. 446 hastanın incelemeleri sonuçlanmıştır, konsey kararı ve ilaç raporları çıkarılmasına rağ men tedavi almadan merkezimizden ayrılmışlardır. Çalışmamızın seçim ve dişlama kriterlerine uygun olacak şekilde ancak 88 hasta kontrol grubu olarak, 32 hasta ise çalışma grubu olarak değerlendirmeye alınmıştır. İstatistiksel verilerde yer alan Grup-1 hastalar kontrol grubunu, Grup-2 hastalar ise çalışma grubunu temsil etmektedir.

İstatistiksel yöntem: Verilerin tanımlayıcı istatistiklerinde ortalama, standart sapma, min-mak, medyan, oran, frekans değerleri kullanılmıştır. Verilerin dağılımına Kolmogorov Simirnov test ile bakılmıştır. Niceliksel verilerin analizinde bağıms1z örneklem t test ve Mann-Whitney u test kullanılmıştır. Niteliksel verilerin analizinde ki-kare test kullanılmıştır. Korelasyon analizinde Spearman ve Pearson korelasyon analizi kullanılmıştır. Analizlerde SPSS 21.0 programı kullanılmıştır.

Tablo 1: İncelenen parametrelerin ortalama değerleri.

\begin{tabular}{|c|c|c|c|c|c|}
\hline & & \multicolumn{2}{|c|}{ Med(Min-Mak) } & \multicolumn{2}{|c|}{ Ort.ts.s./n.\% } \\
\hline \multicolumn{2}{|l|}{ Yaş } & 31 & $20 \cdot 39$ & \multicolumn{2}{|c|}{$31,5 \pm 4,1$} \\
\hline \multicolumn{2}{|l|}{ Infertilite Süresi } & 5 & $2 \cdot 19$ & \multicolumn{2}{|c|}{$6,2 \pm 3,3$} \\
\hline \multicolumn{2}{|l|}{ BMI } & 25 & $18 \cdot 31$ & \multicolumn{2}{|c|}{$24,4 \pm 2,8$} \\
\hline \multicolumn{2}{|l|}{ Bazal FSH } & 8 & $3 \cdot 10$ & \multicolumn{2}{|c|}{$7,5 \pm 1,7$} \\
\hline \multicolumn{2}{|l|}{ Bazal E2 } & 41 & $16 \cdot 356$ & \multicolumn{2}{|c|}{$47,5 \pm 32,8$} \\
\hline \multicolumn{2}{|l|}{ Prolaktin } & 14 & $2 \cdot 38$ & \multicolumn{2}{|c|}{$15,0 \pm 7,0$} \\
\hline \multicolumn{2}{|l|}{ Antral Folikül Sayısı } & 13 & $4 \cdot 31$ & \multicolumn{2}{|c|}{$15,7 \pm 7,2$} \\
\hline \multicolumn{2}{|l|}{ Toplam Gonadotropin Dozu } & 2700 & $1612 \cdot 4975$ & \multicolumn{2}{|c|}{$2869,9 \pm 798,8$} \\
\hline \multicolumn{2}{|l|}{ Toplam Gonadotropin Kullanim Günü } & 9 & $6 \cdot 14$ & \multicolumn{2}{|c|}{$8,9 \pm 1,7$} \\
\hline \multicolumn{2}{|l|}{ Hcg Günü E2 Düzeyi } & 1769 & $285 \cdot 4698$ & \multicolumn{2}{|c|}{$1830,9 \pm 876,4$} \\
\hline \multicolumn{2}{|l|}{ Elde Edilen Total Oosit Sayısı } & 7 & $1 \cdot 15$ & \multicolumn{2}{|c|}{$6,6 \pm 3,1$} \\
\hline \multirow{2}{*}{ Laboratuar Gebelik (Hcg>20) } & Yok & & & 54 & $45,0 \%$ \\
\hline & Var & & & 66 & $55,0 \%$ \\
\hline \multirow{2}{*}{ Klinik Gebelik (+Fetal Kalp Atımı) } & Yok & & & 76 & $63,3 \%$ \\
\hline & Var & & & 44 & $36,7 \%$ \\
\hline
\end{tabular}

Tablo 2: Polikistik over sendromu olan ve olmayan gruplarda ortalama değerler

\begin{tabular}{|c|c|c|c|c|c|c|c|}
\hline \multirow[b]{3}{*}{ Yaş } & \multicolumn{3}{|c|}{ Grupl } & \multicolumn{3}{|c|}{ Grup II } & \multirow{3}{*}{$\frac{p}{0,435}$} \\
\hline & \multirow{2}{*}{$\frac{0 r t . \pm s, s_{1}}{31,6 \pm 4,0}$} & \multicolumn{2}{|c|}{$\operatorname{Med}(\operatorname{Min} \cdot M a k)$} & \multirow{2}{*}{$\frac{0 r t . \pm 5.5 .}{31,0 \pm 4,5}$} & \multicolumn{2}{|c|}{$\operatorname{Med}(\operatorname{Min} \cdot M a k)$} & \\
\hline & & 31 & $23 \cdot 39$ & & 31 & $20 \cdot 38$ & \\
\hline BMI & $24,3 \pm 3,0$ & 25 & $18 \cdot 31$ & $24,8 \pm 2,5$ & 25 & $20 \cdot 29$ & 0,389 \\
\hline Infertilite Süresi & $6,5 \pm 3,5$ & 5 & $2 \cdot 19$ & $5,6 \pm 2,4$ & 5 & $3 \cdot 12$ & 0,292 \\
\hline
\end{tabular}

Tablo 3: Polikistik over sendromu olan ve olmayan hastalarda parametrelerin karşılaştırılması.

\begin{tabular}{|c|c|c|c|c|}
\hline & \multicolumn{2}{|r|}{ Grupl } & \multicolumn{2}{|r|}{ Grupll } \\
\hline & Ort.ts.s.s. & Med(Min-Mak) & Ortits.s.s. & Med(Min-Mak) \\
\hline Bazal FSH & $7,6 \pm 1,6$ & $8 \quad 3 \cdot 10$ & $7,4 \pm 1,7$ & $8 \quad 4 \cdot 10$ \\
\hline Bazal E2 & $50,1 \pm 36,9$ & $42 \quad 20 \cdot 356$ & $40,3 \pm 13,9$ & $41 \quad 16 \cdot 70$ \\
\hline Prolaktin & $15,6 \pm 7,4$ & $14 \quad 2.38$ & $13,3 \pm 5,5$ & $5 \cdot 28$ \\
\hline Antral Folikül Saylsi & $11,9 \pm 3,4$ & $12 \quad 4 \cdot 24$ & $26,5 \pm 3,0$ & $26 \quad 22 \cdot 31$ \\
\hline $\begin{array}{l}\text { Toplam Gonadotropin } \\
\text { Dozu }\end{array}$ & $3054 \pm 806$ & $30001612 \cdot 4975$ & $2340 \pm 483$ & $22501625 \cdot 3150$ \\
\hline $\begin{array}{l}\text { Toplam Gonadotropin } \\
\text { Kullanim Günü }\end{array}$ & $9,0 \pm 1,5$ & $9 \quad 6.13$ & $8,4 \pm 1,9$ & $6 \cdot 14$ \\
\hline Hcg Günü E2 Düzeqi & $1838 \pm 940$ & $1761285 \cdot 4698$ & $1810 \pm 673$ & $1788875 \cdot 3451$ \\
\hline Oosit Saysis & $6,2 \pm 3,3$ & $1 \cdot 15$ & $7,7 \pm 2,0$ & $3 \cdot 11$ \\
\hline
\end{tabular}


Tablo 4: Polikistik over sendromu olan ve olmayan gruplarda gebelik sonuçları.

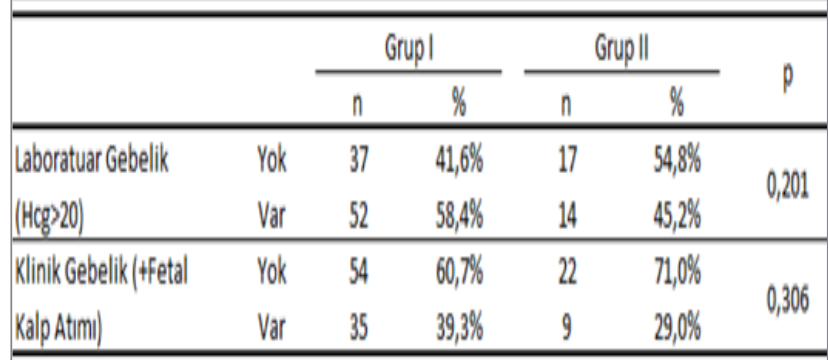

Ki-kare test

Tablo 5: Polikistik over sendromu olan grupta antral folikül sayısı ile diğer parametrelerin iliş̧isi.

\begin{tabular}{|c|c|c|c|c|c|c|}
\hline Grup II & & Yaş & $\begin{array}{c}\text { Infertilite } \\
\text { Süresi }\end{array}$ & BMI & Bazal Fsh & Bazal E2 \\
\hline Antral Folikül & $r$ & $\cdot 0,034$ & 0,022 & $-0,442$ & 0,179 & $.0,168$ \\
\hline \multirow[t]{2}{*}{ Say|s| } & $p$ & 0,857 & 0,908 & 0,013 & 0,336 & 0,367 \\
\hline & & Prolaktin & $\begin{array}{c}\text { Toplam } \\
\text { Gonadotropin } \\
\text { Dozu }\end{array}$ & $\begin{array}{c}\text { Toplam } \\
\text { Gonadotropin } \\
\text { Kullanım Günü }\end{array}$ & $\begin{array}{c}\text { Hcg Günü E2 } \\
\text { Düzevi }\end{array}$ & $\begin{array}{c}\text { Elde Edilen } \\
\text { Total Oosit } \\
\text { Say|si }\end{array}$ \\
\hline Antral Folikül & r & 0,063 & $\cdot 0,190$ & 0,054 & 0,390 & 0,171 \\
\hline Sayls! & $p$ & 0,737 & 0,306 & 0,774 & 0,030 & 0,357 \\
\hline
\end{tabular}

\section{Çalışmamızın yapılan veri analizinde;}

Polikistik over sendromu tanısı olan (Grup-2) ve olmayan gruplar (Grup-1) karşılaştırıldığında; yas aralıkları, infertilite süreleri, beden kitle indeksleri açısından anlamlı fark saptanmamıştır (Tablo 2-3). Grup 1 ve 2 hastalarm bazal FSH, E2, prolaktin ve hCG günü E2 değerleri açısından anlamlı fark saptanmamıștır (Tablo-2). İstatistiksel olarak anlamlı şekilde polikistik over sendromlu grupta kullanılan total gonadotropin dozu ve kullanılan gün sayısı daha düşük saptanmıștır. Toplam elde edilen oosit sayıs1 PKOS grubunda anlamlı olarak yüksek bulunmuştur ancak bu sonuçlar laboratuar ve klinik gebelik sonuçlarına yansımamıştır (Tablo 3-4). Polikistik over sendromu olmayan grupta antral follikül sayısı ile diğer parametrelerin (yaş, infertilite süresi, beden kitle indeksi) arasında korelasyon bulunmamıştır (Tablo-5) Ancak antral follikül sayısı ile kullanılan total gonadotropin düzeyi arasında istatistiksel olarak anlamlı düzeyde negatif korelasyon bulunmuştur (Tablo-5). Son olarak; antral follikül sayısının klinik ve laboratuar gebelik oranlarıyla korelasyonu görülmemiştir.

\section{TARTIŞMA}

Çalışmamızın yapılan veri analizine göre; polikistik over sendromu tanıs1 olan (Grup-2) ve olmayan gruplar (Grup-1) arasında; yaş aralıkları, infertilite süreleri, beden kitle indeksleri açısından fark bulunamamıștır. Polikistik over sendromu olan ve olmayan hastalarda bazal FSH, E2, prolaktin ve hCG günü E2 değerleri açısından fark saptanmamıştır. Polikistik over sendromu olmayan grupta antral follikül sayısı ile diğer parametrelerin (yaş, inferti- lite süresi, beden kitle indeksi) arasında korelasyon bulunmamasına rağmen, antral follikül sayısı ile kullanılan toplam gonadotropin düzeyi arasındaki istatistiksel anlamlı düzeyde negatif korelasyon bulunması öncelikli bulgularımız arasındaydı. Diğer yandan polikistik over sendromlu grupta kullanilan total gonadotropin dozu ve kullanılan gün sayısının düşük saptanması genel literatür bilgisiyle uyumlu bulunmuştur. Toplam elde edilen oosit sayısı PKOS grubunda anlamlı şekilde yüksek bulunmasına rağmen, elde edilen sonuçlar laboratuvar ve klinik gebelik sonuçlarına yansımamıştır. Bunun da temel sebebinin; güncel yayınlarda da belirtildiği gibi, polikistik over sendromunun metabolik ve hormonal etkilerinin oosit kalitesi ve döllenme üzerine olan olumsuz etkileri nedeniyle olduğu kanaatindeyiz (5). Çalışmamızın dizaynı ve analiz sonuçlarına da bakarak literatür incelendiği zaman çalıșmamızla birebir olarak örtüșen çalıșmalara rastlayamadık. Ancak çalıșmamızdaki parametrelerle yakından ilişkide bulunan ve çalışmamıza da destek oluşturan polikistik over sendromu ile Gnrh-antagonist ve agonist protokolleri içeren karşılaştırmalı çalışmalarla ilgili literatürü gözden geçirdik.

Diedrich ve ark.'ları yaptıkları çalışmada; önceki tedavi sikluslarında erken LH piki nedeniyle siklus iptali yaşanmıș, ve tedavi olarak $\mathrm{KOH}$ planlanan hastalarda Gnrh antagonisti (cetroreliks) kullanarak sonuçlarını benzer demografik ve hormonal özelliklere sahip hastaların uzun protokol Gnrh agonisti kullanılmış tedavi sikluslarıyla karşılaştırmışlardır. Hastalara adetin 2. günü hMG ile $\mathrm{KOH}$ başlanmış, ve stimulasyonun 7. gününden itibaren cetroreliks $0.25 \mathrm{mgr} /$ gün eklenmiştir. Antagonist uygulanan hastalarda, agonist sikluslariyla kiyaslandığında, daha kısa süreli stimulasyon ve daha az miktarda gonadotropin kullanılmıştır ancak IVF sonuçları benzer bulunmuştur. Araştırmacılar, özellikle erken LH piki nedeniyle siklus iptali yaşanmış hastalarda, gonadotropinlere eklenen Gnrh antagonisti kullanımının alternatif bir tedavi seçeneği olabileceğini belirtmişlerdir (6).

Borm ve Mannaerts, Avrupa Orgalutran Çalışma Grubunun yaptığı çok merkezli prospektif kontrollü araştırma sonuçlarını, yayımladıkları makalelerinde yorumlamışlardır. Bu çalışmada, yardımeı üreme tekniği amaciyla r-FSH (Puregon) ile $\mathrm{KOH}$ uygulanan hastalarda Gnrh antagonisti (ganireliks) ile Gnrh agonisti (buserelin, intranasal) sonuçlar1 karşılaştırılmıştır. Fertilizasyon oranları benzer bulunurken, implantasyon oranları ganirelix ve buserelin için sırasıyla \%15.7 ve \%21.8 saptanmıştır. Devam eden gebelik oranları ise sirasiyla $\% 20.3$ ve \%25.7 saptanmıştır. Araştırmacılar, bu çalışma sonucunda ganireliks içeren tedavi protokollerinin, YÜT amaciyla $\mathrm{KOH}$ uygulanacak hastalarda etkili, kısa ve güvenilir olduğu sonucuna varmışlardır (7).

Fluker ve ark.'ları IVF planlanan 313 hastada, Gnrh antagonisti (Cetrorelix) ile Gnrh agonistini (leuprolide acetate) karşılaştırmalı olarak değerlendirmişlerdir. Matür oosit sayısı antagonist grubunda 11.6, agonist grubunda 14.1 saptanmıştır. Fertilizasyon oranları sirasiyla \%62.4 ve \%61.9, implantasyon oranlar1 \%21.1 ve \%26.1, klinik gebelik oranlar1 $\% 35.4$ ve $\% 38.4$, devam eden gebelik oranları 
ise $\% 30.8$ ve $\% 36.4$ olarak bulunmuştur (8).Çalışmanın analizinde; Gnrh antagonist grupta daha az enjeksiyon miktarı ve daha az uygulama süresiyle benzer gebelik oranları belirtilmiş ve agonist gruba göre daha az yan etki saptanmıştır.

Lambalk ve ark. Yaptığ metaanalizde PKOS'lu hastalarda Gnrh antagonist kullanılan grupta uzun protokol agonist grubuna göre OHSS daha az gözlenmiştir. Klinik gebelik oranlarında fark görülmemiştir.yine aynı çalışmada genel IVF grubunda (PKOS olmayan) antagonist protokol uygulananlarda uzun agonist protokole göre toplanan oosit sayısı ve gebelik oranları düşük saptanmıştır. Temel sebepler olarak yetersiz LH piki baskılanmaS1 ve senkron follikül gelişimi olmaması şeklinde açıklanmıştır. PKOS grubunda ise farklı siklus dinamikleri olduğu için steriod olmaksızın tek başına gnrh analog kullanmak yeterli değildir (9).

Griesinger ve ark. Yaptı $\breve{g}_{1}$ metaanalizde; PKOS'lu hastalarda Gnrh agonist ve antagonist protokollerde toplanan oosit sayısında fark görülmemiştir (10). Ancak, Trankic ve ark. Yaptığı çalışmada, genel literatürle uyumlu olarak, agonist kullanılan grupta toplanan oosit sayısı fazla saptanmiştır (11).

\section{SONUC}

Polikistik over sendromu infertil hastalar içinde önemli yer tutmaktadır. Hastalığın hem metabolik, hem de endokrinolojik özellikleri göz önüne alınarak fertilite üzerine olan olumsuz özellikleri nedeniyle kontrollü over stimulasyonu ve IVF planlaması özel önem taşımaktadır.

Çalışmamızda polikistik over sendromu tanısı alan infertil hastalarda $\mathrm{KOH}$ protokolü uygulaması sonucu istatistiksel anlaml şekilde $(\mathrm{p}<0,05)$ daha fazla oosit elde edilmiştir. Ancak gebelik sonuçlarının farklılık göstermemiştir.Literatürde de belirtildiği gibi polikistik over sendromunda görülen metabolik ve hormonal açıdan bozulmuş moleküler mekanizmaların oosit kalitesi ve fertilizasyon yeteneğinin bozulmasında önemli rolü vardır. Endometrial reseptivitenin de olumsuz etkilediğini görüşü ağırlık kazanmaktadır. Bu konudaki yapılacak moleküler çalışmaların etiyoloji ve patogeneze katkı sunacağ aşikardir.

Çalışmamızda belirtilen kriterlere göre belirlenmiş hastaların sınırlı sayıda olması çalışmamızın kısıtlllıkları arasında sayılabilir. Bu nedenle daha geniş vaka sayılarıyla oluşturulmuş çalışmalara ihtiyaç vardır.

\section{KAYNAKLAR}

1. Azziz R, Woods KS, Reyna K, Key TJ, Knochenhauer ES, Yildiz BO. The prevalence and features of the polycystic ovary syndrome in an unselected population. J Clin Endocrinol Metab 2004; 89:2745-9.

2. Yildiz BO, Chang W, Azziz R. Polycystic ovary syndrome and ovulation induction. Minerva Ginecol 2003; 55:425-39

3. Adashi EY, Rock JA, Resonwacks Z, Reproductive endocrinology surgery and technology. 1996; chapter60: 1208-1223

4. Macklon NS, Fauser BCJM. Progress in ovarian stimulation. Ann. d'Endocrinol 1999; 60: 137-142.

5. Speroff L, Marc A. Fritz; Clinical Gyneacologic Endocrinology and İnfertility. Williams \&Wilkins, Baltimore. 8. Edition,2010; 256-257

6. Diedrich K, Diedrich C, Santos E, Zoll C, al-Hasani S; Suppression of the endogenous luteinizing hormone surge by the gonadotrophin-releasing hormone antagonist Cetrorelix during ovarian stimulation; Hum Reprod. 1994 May;9(5):788-91.

7. Borm G, Mannaerts B; Treatment with the gonadotrophin-releasing hormone antagonist ganirelix in women undergoing ovarian stimulation with recombinant follicle stimulating hormone is effective, safe and convenient: results of a controlled, randomized, multicentre trial. The European Orgalutran Study Group; Hum Reprod. 2000 Jul;15(7):1490-8

8. Fluker M, Grifo J, Leader A; Efficacy and safety of ganirelix acetate versus leuprolide acetate in undergoing controlled ovarian hyperstimulation; Fertil Steril. 2001 Jan;75(1):38-45

9. Lambalk CB, Banga FR, Huirne JA; GnRH antagonist versus long agonist protocols in IVF: a systematic review and meta-analysis accounting for patient type; Human Reproduction Update, Volume 23, Issue 5, 1 September 2017, Pages 560-579.

10. Griesinger G, Diedrich K, Tarlatzis BC, [et al.]. GnRH-antagonists in ovarian stimulation for IVF in patients with poor response to gonadotrophins, polycystic ovary syndrome, and risk of ovarian hyperstimulation: A meta-analysis. Reprod BioMed Online. 2006, 13 (5), 628-638.

11. Milan Trenkić, Jasmina Popović, Vesna Kopitović, Artur Bjelica, Radomir Živadinović, Sonja Pop-Trajković. Flexible $\mathrm{GnRH}$ antagonist protocol vs. long GnRH agonist protocol in patients with polycystic ovary syndrome treated for IVF: comparison of clinical outcome and embryo quality. Ginekol Pol 2016;87(4):265-270. 